\title{
Intervención basada en el modelo de solución de problemas para cuidadores de enfermos renales crónicos
}

\author{
Intervention based on the problem-solving model \\ for caregivers of chronic kidney disease patients
}

\author{
Karla Nathalia Fernández Castillo ${ }^{1}$, Teresa Iveth Sotelo Quiñonez ${ }^{2}$, \\ Raquel García Flores ${ }^{2}$, Nora Hemi Campos Rivera ${ }^{2}$ \\ y Santa Magdalena Mercado Ibarra ${ }^{2}$
}

\begin{abstract}
RESUMEN
Introducción: Los cuidadores de enfermos renales crónicos ponen en riesgo su salud física y mental debido al estrés constante relacionado con el cuidado de este tipo de pacientes. Objetivo: Evaluar los efectos de un programa de intervención basado en el modelo de solución de problemas sobre la percepción de sobrecarga, ansiedad y depresión en cuidadores informales de pacientes con enfermedad renal crónica. Método: Se desarrolló un estudio cuasiexperimental de dos grupos (control en lista de espera y experimental), con pretest y postest, utilizando para ello los inventarios de Ansiedad y Depresión de Beck y la Escala de Sobrecarga del Cuidador. Participaron 21 cuidadores informales, familiares de pacientes con enfermedad renal crónica. Resultados: Se encontraron efectos significativos en la prueba de Wilcoxon al final del tratamiento y al mes de seguimiento en la sintomatología depresiva y ansiosa y la percepción de sobrecarga en el grupo experimental. El tamaño del efecto $d$ de Cohen fue grande en depresión y sobrecarga y mediano en ansiedad, y se identificó una disminución grande de todas las variables. En el grupo control no se hallaron diferencias. Discusión: La intervención basada en el modelo de solución de problemas fue eficaz para disminuir la sintomatología depresiva y ansiosa, y la percepción de sobrecarga en los cuidadores primarios de enfermos renales crónicos.
\end{abstract}

Palabras clave: Cuidadores; Enfermedad renal crónica; Modelo de solución de problemas; Terapia cognitivo-conductual; Sobrecarga.

\begin{abstract}
Introduction: Caregivers of chronic kidney disease (CKD) patients are at risk for physical and mental health problems due to the constant stress associated with caring for these patients. Objective: To evaluate the effects of an intervention based on the problem-solving model on the perception of overload, anxiety and depression in informal caregivers of patients with CKD. Method: The study involved a quasi-experimental study of two groups (waiting list and experimental), with pretest and post-test measurements. Measures included Beck's anxiety and depression inventories and an overload caregiver scale. Participants were 21 informal caregivers of patients with chronic kidney disease. Results: Significant improvements were found through the Wilcoxon's test at the end of the treatment and at one month follow-up in the depressive, anxious and overload perception symptoms in the experimental group. Results on the effect size d of Cohen was "large" in depression and overload, "medium" in anxiety. A "large" improvement in other variables was identified. No differences
\end{abstract}

\footnotetext{
${ }^{1}$ Departamento de Psicología y Ciencias de la Comunicación, Universidad de Sonora, Boulevard Luis Encinas y Rosales, Col. Centro, 83000 Hermosillo, Son., México, tel. (644)110-13-43, correo electrónico: nath_fercas@hotmail.com. Artículo recibido el 15 de agosto y aceptado el 13 de noviembre de 2017.

${ }^{2}$ Departamento de Psicología, Instituto Tecnológico de Sonora, 5 de Febrero 818 Sur, Col. Centro, 85000 Ciudad Obregón, Son., México, tel. (644)410-09-01, exts.2458,2457,2460y 2455, correos electrónicos: teresa.sotelo@itson.edu.mx, raquel.garcia@itson.edu.mx, nora.campos@ itson.edu.mx y mmercado@itson.edu.mx.

Citación: Fernández C., K.N., Sotelo Q., T.I., García F., R., Campos R., N.H. y Mercado I., S.M. (2018). Intervención basada en el modelo de solución de problemas para cuidadores de enfermos renales crónicos. Psicología y Salud, 28(2), 251-259.
} 
were identified in the control group. Discussion: The intervention based on the problem-solving model was effective in reducing the depressive, anxious symptomatology and perceived overload of the primary caregivers of chronic renal patients.

Key words: Caregivers; Kidney chronical disease; Problem solving model; Cognitive-behavioral therapy; Overload.

\section{INTRODUCCIÓN}

$\mathrm{L}$

as enfermedades crónico-degenerativas son la principal causa de muerte en México y en el mundo (Instituto Nacional de Estadística y Geografía, 2013; Organización Mundial de la Salud, 2017); su larga duración y progresión lenta provoca dependencia total o parcial en las personas que las padecen. La enfermedad renal crónica (ERC en lo sucesivo) afecta a mexicanos de todas las edades, aunque hay una alta prevalencia en adultos mayores que la padecen, además de otras enfermedades como la diabetes y la hipertensión. En un estudio realizado por Amato et al. (2005) se calculó que la prevalencia de la depuración de creatinina (un indicador de ERC) menor a $15 \mathrm{ml} /$ min fue de 1,142 por millón de habitantes. Los factores asociados a este resultado fueron el consumo de tabaco y alcohol, el género femenino, una edad mayor a 65 años, una escolaridad menor al nivel primario e ingresos económicos menores a cuatro dólares diarios. Tales datos son semejantes a los que se han reportado en los países industrializados.

La característica de esta enfermedad es que las personas que la sufren dependen en las últimas etapas de tratamientos sustitutivos de la función renal, como la diálisis o la hemodiálisis. Estos tratamientos afectan la calidad de vida de las personas enfermas porque deben hacer cambios importantes en su estilo de vida, tales como en la alimentación, la ingesta de líquidos y las actividades laborales y sociales, entre muchos otros (Borroto, Almeida, Lorenzo, Alfonso y Guerrero, 2007).

$\mathrm{Al}$ afectarse la independencia y la autonomía de las personas con ERC, estas requieren la ayuda de otras personas para subsistir, que en muchos casos son miembros de la familia, en los que recae toda la responsabilidad del cuidado. El familiar apoya al enfermo en las actividades cotidianas, identifica sus necesidades, participa en la toma de decisiones y verifica el desarrollo de acciones como la toma de medicamentos, los cambios de hábitos alimenticios o el fomento de la actividad física. Además de motivarlo y apoyarlo emocionalmente, el cuidador también acompaña al enfermo a las visitas rutinarias a los centros asistenciales y a los exámenes de control (Bustos, 2006; De la Cuesta, 2004; Fernández, 2006). Es frecuente que el cuidador principal, en muchos casos familiar, lleve a cabo estas actividades sin recibir pago económico alguno ni capacitación previa para la atención del enfermo (Armstrong, 2005). Por ende, la función del cuidador principal es esencial para la supervivencia del enfermo; sin embargo, pone en riesgo su salud física y mental debido a que se ve sometido a un estrés constante al alterar su rol habitual para brindar cuidados a la persona enferma; por esta razón, debe hacer constantes ajustes a sus actividades laborales, de ocio y de esparcimiento, entre otras (Roca et al., 2000). Además, debe soportar la severidad de la enfermedad del receptor del cuidado, su posible pérdida y la falta de apoyo de los otros miembros de la familia (Flórez, Montalvo, Herrera y Romero, 2010).

A menudo el familiar que cuida al enfermo desconoce por completo el riesgo que implica desempeñar ese rol durante un tiempo prolongado (Seidmann, Bail, Acrich y Stéfani, 2001), pues la labor diaria y el largo plazo -así se asuma de manera voluntaria y con afecto- puede traer como consecuencia diversos riesgos para la salud, sobre todo si la responsabilidad recae por entero en una sola persona (Centro Nacional de Equidad de Género y Salud Reproductiva de la Secretaría de Salud y Centro Oncológico Integral Diana Laura Riojas de Colosio del Hospital Médica Sur, 2006). Algunos de los padecimientos que tiende a desarrollar el familiar cuidador son los trastornos del sueño, ansiedad, depresión, irritabilidad, sentimientos de carga y de desesperanza, resentimiento hacia la persona cuidada, pensamientos de suicidio y sentimientos de abandono o soledad, cefaleas, cansancio y aislamiento (Zambrano y Ceballos, 2007). Por lo tanto, es importante que se les ofrezca el apoyo necesario para que tengan 
una vida digna y sigan brindando al enfermo la ayuda que requiere (Archury, Castaño, Gómez y Guevara, 2011).

Varios programas de intervención benefician al cuidador de personas con enfermedades crónicas como la demencia (Ducharme et al., 2011; Espín, 2009, 2011; Losada y Montorio, 2005; Nobili et al., 2004; Vinyoles et al., 2001) y diversos tipos de cáncer (Ko et al., 2005; Meyers et al., 2011; Montero et al., 2012; Rangel et al., 2013), entre otras. Las intervenciones difieren en el tipo de estrategias de intervención; por ejemplo, programas psicoeducativos (Bañobre et al., 2005; Ducharme et al., 2011; Espín, 2009, 2011; Rangel et al., 2013; Velásquez, López, López, Cataño y Muñoz, 2011), psicoterapéuticos (Montero et al., 2012), de habilidades para el cuidado (Barrera et al., 2006; Vega, Mendoza, Ureña y Villamil, 2008) o de apoyo domiciliario (Guerrero et al., 2008; Nobili et al., 2004). Todas estas intervenciones no siempre se aplican de forma única o aislada, sino que en ocasiones se desarrollan al mismo tiempo y en combinaciones muy variadas (Martín, Ballesteros, Domínguez, Muñoz y Gonzáles, 2014). También se interviene en la modalidad grupal (Espín, 2009; Velásquez et al., 2011) e individual (Rangel et al., 2013), con diferentes objetivos, como reducir la depresión (Otero, Vázquez, Ferraces, Blanco y Torres, 2014; Vázquez, Otero, López, Blanco y Torres, 2010) y la ansiedad (Ferré et al., 2012), incrementar las habilidades para afrontar las demandas del cuidado (Cameron, Shin, Williams y Stewart, 2004) y la calidad de vida (Demiris et al., 2010), y finalmente la adquisición de habilidades de autocuidado (Rangel et al., 2013).

El entrenamiento en el modelo de solución de problemas es una de las técnicas de intervención psicológica que se aplica a cuidadores informales de pacientes con enfermedades crónicas diversas (Elliott, Berry y Grant, 2009; Meyers et al., 2011; Rangel et al., 2013; Sherwood et al., 2012; Tong, Yip, Liu y McMaster, 2016; Vázquez et al., 2010; Vázquez et al., 2013).

Debido a que la solución de problemas implica un proceso cognitivo y comportamental que ayuda a las personas a tener disponibles alternativas de respuesta variadas para encarar situaciones problemáticas; en los cuidadores son útiles para afrontar el cuidado y las demandas de otros roles, como trabajar, cuidar del hogar o a otros miem- bros de la familia, y a la vez aumentar la probabilidad de elegir las respuestas más eficaces entre las alternativas posibles.

Una de las ventajas del uso de esta estrategia es que se utiliza para prevenir problemas y para fomentar la autonomía (D'Zurilla, 1993). Por lo tanto, el objetivo del estudio cuasiexperimental, con dos grupos (control en lista de espera y experimental), con pretest y postest, sin aleatorización, fue evaluar los efectos de un programa de intervención basado en el modelo de solución de problemas sobre la percepción de sobrecarga, ansiedad y depresión en cuidadores informales de pacientes con ERC.

\section{MÉTODO}

\section{Participantes}

Participaron 21 cuidadores informales, familiares de pacientes con ERC. De ellos, $85.7 \%$ eran mujeres y $14.3 \%$ hombres, con una media de edad de 44 años, con rango de 20 a 62 . Respecto al parentesco, $33 \%$ eran las hijas y $28 \%$ las esposas de los pacientes y convivían con estos. Los criterios de inclusión fueron que el participante tuviera a cargo el cuidado principal y que no recibiera una remuneración económica por sus servicios. Si no cumplían con estas condiciones, se les excluía del estudio.

\section{Instrumentos}

Inventario de Ansiedad de Beck (BAI) (Beck y Steer, 1993a). Estandarizado en población mexicana por Robles, Varela, Jurado y Páez (2001), está conformado por 21 reactivos que miden sintomatología ansiosa (por ejemplo: miedo a perder el control), con una escala Likert de cuatro opciones de respuesta que van de 0 (poco o nada) a 3 (severamente). Posee un coeficiente alfa de Cronbach de .83 y una validez de constructo de cuatro factores principales, congruentes con los referidos por los autores de la versión original: subjetivo, neurofisiológico, autonómico y pánico. Con una confiabilidad test-retest de .75 y una validez convergente entre el Inventario de Beck de Ansiedad y el Inventario de Ansiedad Rasgo-Estado (IDARE) (Spielberger y Díaz-Guerrero, 1975), muestran ín- 
dices de correlación moderados y positivos y validez discriminante que permite detectar pacientes con trastornos de ansiedad y normales $(\mathrm{t}=-19.11$, $p<.05)$.

Inventario de Depresión de Beck (IDB) (Beck y Steer, 1993b). Se utilizó la adaptación hecha por Jurado et al. (1998) para población mexicana. Esta versión consta de 21 reactivos (por ejemplo, "Me siento triste todo el tiempo") que se responde con un escalograma de Guttman con cuatro opciones de respuesta que van de menor a mayor intensidad: 0 ("Yo no me siento triste"), 1 ("Me siento triste"), 2 ("Me siento triste todo el tiempo"), 3 ("Estoy tan triste, tan infeliz, que no puedo soportarlo").

Escala de Sobrecarga del Cuidador. Desarrollada por Domínguez (2012). Consta de diez reactivos que miden la frecuencia con la que el participante percibe haber tenido consecuencias negativas en diferentes áreas de la vida, como la salud física y la emocional, las relaciones sociales, el tiempo y los planes personales (por ejemplo, cambiar proyectos o metas personales). Se contesta con una escala tipo Likert de cuatro puntos con recorrido de 0 ("Nunca") a 3 ("Siempre"). La validación de la escala en cuidadores hermosillenses indicó que es unidimensional y que explica 38\% de varianza del constructo, registrándose un valor de consistencia interna de .85 .

\section{Procedimiento}

Se estableció contacto con la institución para tener acceso a las instalaciones. Tras la autorización correspondiente, se invitó a los cuidadores principales de los pacientes con ERC que se encontraran en la sala de espera a que participaran en el estudio. Una vez que aceptaban, se daba lectura al formato de consentimiento informado, donde se daban a conocer los objetivos del estudio, para que lo firmaran. Se aplicó como medición en el pretest los instrumentos antes mencionados, dividiendo a los participantes según el horario en que tenían la sesión de hemodiálisis. Un grupo de participantes se mantuvo en lista de espera. Se aplicó el programa de intervención de forma grupal con el objetivo de instruir a los cuidadores de pacientes con insuficiencia renal crónica para que identificaran y aplicaran los pasos del modelo de solución de problemas en las dimensiones económicas, sociales, personales y problemáticas relacionadas al cuidado, con base en las instrucciones precisas del manual del instructor y del participante (Tabla 1). El programa de intervención basado en el citado modelo consta de nueve sesiones grupales de una hora y media cada una. Finalizado el programa, se aplicaron los mismos instrumentos como medición postest. Dicha medición se aplicó también al grupo en lista de espera. Se llevó a cabo la misma intervención en el grupo en lista de espera y similares mediciones posteriores. Se realizaron dos seguimientos: uno al mes y otro a los dos meses de terminada la intervención. Para el análisis de los datos se utilizaron estadísticos descriptivos no paramétricos, en particular la prueba de Wilcoxon, y para calcular el tamaño del efecto se usó la $d$ de Cohen mediante el software SPSS, versión 21.

Tabla 1. Sesiones, contenidos y actividades del programa de intervención.

\begin{tabular}{|c|c|c|}
\hline SESIÓN & CONTENIDOS & ACTIVIDADES \\
\hline \multirow{4}{*}{1} & Bienvenida y presentación. & \\
\hline & $\begin{array}{l}\text { Objetivo del programa y explicación } \\
\text { del formato de consentimiento informado. }\end{array}$ & $\begin{array}{l}\text { Identificar el objetivo general del programa. } \\
\text { Analizar y firmar el formato de consentimiento informado. }\end{array}$ \\
\hline & $\begin{array}{l}\text { Aplicación de instrumentos de medición } \\
\text { pretest. }\end{array}$ & Realizar la evaluación pretest. \\
\hline & $\begin{array}{l}\text { Descripción de los contenidos } \\
\text { del programa. }\end{array}$ & Identificar los contenidos del programa. \\
\hline \multirow[b]{2}{*}{2} & $\begin{array}{l}\text { Identificación y normalización } \\
\text { del problema. }\end{array}$ & $\begin{array}{l}\text { Identificar los objetivos y fases del modelo de solución } \\
\text { de problemas. }\end{array}$ \\
\hline & $\begin{array}{l}\text { Descripción de pasos para la solución } \\
\text { del problema. }\end{array}$ & $\begin{array}{l}\text { Discutir sobre la definición de un problema. } \\
\text { Discutir sobre qué es la solución de un problema. } \\
\text { Explicar los cinco pasos para la solución de un problema. } \\
\text { Discriminar problemas de una lista de situaciones. }\end{array}$ \\
\hline
\end{tabular}




\begin{tabular}{|c|c|c|}
\hline 3 & $\begin{array}{l}\text { Aplicación del modelo de solución } \\
\text { de problemas a un caso simulado } \\
\text { relacionado con el estrés del cuidado. }\end{array}$ & $\begin{array}{l}\text { Resumir la sesión anterior y aclarar dudas. } \\
\text { Identificar el problema en el caso simulado. } \\
\text { Identificar las emociones. } \\
\text { Realizar una lluvia de ideas de las alternativas de solución. } \\
\text { Analizar las ventajas y desventajas de las alternativas } \\
\text { de solución. } \\
\text { Elegir una alternativa de solución. } \\
\text { Planear la implementación de la solución y visualizar } \\
\text { los posibles resultados. }\end{array}$ \\
\hline 4 & $\begin{array}{l}\text { Aplicación del modelo de solución } \\
\text { de problemas a un caso simulado } \\
\text { relacionado con problemas económicos } \\
\text { y de transporte. }\end{array}$ & $\begin{array}{l}\text { Resumir la sesión anterior y aclarar dudas. } \\
\text { Identificar el problema en el caso simulado. } \\
\text { Identificar las emociones. } \\
\text { Realizar una lluvia de ideas sobre las alternativas de solución. } \\
\text { Analizar las ventajas y desventajas de las alternativas } \\
\text { de solución. } \\
\text { Elegir una alternativa de solución. } \\
\text { Planear la implementación de la solución y visualizar } \\
\text { los posibles resultados. }\end{array}$ \\
\hline 5 & $\begin{array}{l}\text { Aplicación del modelo de solución } \\
\text { de problemas a un caso simulado } \\
\text { relacionado con problemas emocionales } \\
\text { en el paciente. }\end{array}$ & $\begin{array}{l}\text { Resumir la sesión anterior y aclarar dudas. } \\
\text { Identificar el problema en el caso simulado. } \\
\text { Identificar las emociones. } \\
\text { Realizar una lluvia de ideas sobre las alternativas de solución. } \\
\text { Analizar las ventajas y desventajas de las alternativas } \\
\text { de solución. } \\
\text { Elegir una alternativa de solución. } \\
\text { Planear la implementación de la solución y visualizar } \\
\text { los posibles resultados. }\end{array}$ \\
\hline 6 & $\begin{array}{l}\text { Aplicación del modelo de solución } \\
\text { de problemas a un caso simulado } \\
\text { relacionado con los problemas emocionales } \\
\text { del cuidador. }\end{array}$ & $\begin{array}{l}\text { Resumir la sesión anterior y aclarar dudas. } \\
\text { Identificar el problema en el caso simulado. } \\
\text { Identificar las emociones. } \\
\text { Realizar una lluvia de ideas sobre las alternativas de solución. } \\
\text { Analizar las ventajas y desventajas de las alternativas } \\
\text { de solución. } \\
\text { Elegir una alternativa de solución. } \\
\text { Planear la implementación de la solución y visualizar } \\
\text { los posibles resultados. }\end{array}$ \\
\hline 7 & $\begin{array}{l}\text { Aplicación del modelo de solución } \\
\text { de problemas a un caso simulado } \\
\text { relacionado con el área social del cuidador. }\end{array}$ & $\begin{array}{l}\text { Resumir la sesión anterior y aclarar dudas. } \\
\text { Identificar el problema en el caso simulado. } \\
\text { Identificar las emociones. } \\
\text { Realizar una lluvia de ideas sobre las alternativas de solución. } \\
\text { Analizar las ventajas y desventajas de las alternativas } \\
\text { de solución. } \\
\text { Elegir una alternativa de solución. } \\
\text { Planear la implementación de la solución y visualizar } \\
\text { los posibles resultados. }\end{array}$ \\
\hline 8 & $\begin{array}{l}\text { Aplicación del modelo de solución } \\
\text { de problemas a una problemática propia } \\
\text { del participante. }\end{array}$ & $\begin{array}{l}\text { Resumir la sesión anterior y aclarar dudas. } \\
\text { Identificar el problema en el caso simulado. } \\
\text { Identificar las emociones. } \\
\text { Realizar una lluvia de ideas sobre las alternativas de solución. } \\
\text { Analizar las ventajas y desventajas de las alternativas } \\
\text { de solución. } \\
\text { Elegir una alternativa de solución. } \\
\text { Planear la implementación de la solución y visualizar } \\
\text { los posibles resultados. }\end{array}$ \\
\hline 9 & $\begin{array}{l}\text { Aplicación de las pruebas de postest, } \\
\text { retroalimentando las diferentes sesiones } \\
\text { llevadas a cabo con anterioridad. }\end{array}$ & $\begin{array}{l}\text { Resumir la sesión anterior. } \\
\text { Retroalimentar los resultados y aclarar dudas. } \\
\text { Resumir los beneficios obtenidos en el taller. } \\
\text { Contestar la evaluación postest. } \\
\text { Agradecer la participación, y cierre del programa. }\end{array}$ \\
\hline
\end{tabular}




\section{RESULTADOS}

Se llevó a cabo un análisis univariado para el puntaje total de la ansiedad, depresión y sobrecarga en el pretest, postest y seguimientos 1 y 2 . Al comparar las medidas de tendencia central entre el pretest y las medidas subsecuentes, se observa la tendencia a disminuir en las variables de ansiedad, depresión y sobrecarga (Tabla 2).

Tabla 2. Estadística univariada del puntaje total de las variables en el pretest, postest y seguimientos 1 y 2.

\begin{tabular}{|c|c|c|c|c|c|c|c|c|c|c|c|}
\hline \multirow{3}{*}{$\begin{array}{l}\text { Medidas } \\
\text { descriptivas }\end{array}$} & \multicolumn{11}{|c|}{ Variables $(n=21)$} \\
\hline & \multicolumn{4}{|c|}{ Ansiedad } & \multicolumn{4}{|c|}{ Depresión } & \multicolumn{3}{|c|}{ Sobrecarga } \\
\hline & Pre & Post & Seg1 & Seg2 & Pre & Post & Seg1 & Seg2 & Pre & Pos & Seg1 \\
\hline Media & 18.9 & 13.1 & 13.5 & 8.7 & 34.7 & 25.2 & 22.2 & 18.3 & 21.2 & 15.5 & 12.1 \\
\hline Mediana & 17.0 & 9.0 & 12 & 8 & 30 & 24 & 22 & 19 & 21 & 15 & 12 \\
\hline Moda & 7 & 5 & 0 & 0 & 22 & 21 & 20 & 19 & 15 & 17 & 6 \\
\hline D.E. & 11.7 & 10.2 & 11 & 8 & 11.3 & 5.2 & 6.4 & 5.8 & 6.6 & 5.9 & 5.2 \\
\hline Valor mín. & 6 & 1 & 0 & 0 & 22 & 18 & 10 & 5 & 10 & 7 & 5 \\
\hline Valor máx. & 42 & 35 & 34 & 23 & 58 & 38 & 33 & 28 & 40 & 30 & 22 \\
\hline
\end{tabular}

Se aplicó la prueba de rangos de Wilcoxon entre las puntuaciones pretest-postest, preseguimiento 1 y preseguimiento 2 de los grupos de intervención y control, encontrándose datos significativos en el grupo experimental en las variables de ansiedad, depresión y sobrecarga. En el grupo control (lista de espera) no se hallaron diferencias significativas (Tabla 3).

Tabla 3. Prueba de Wilcoxon entre el pretest, postest, seguimientos y grupo control.

\begin{tabular}{|c|c|c|c|c|c|}
\hline \multirow{2}{*}{ Escalas } & & \multicolumn{3}{|c|}{ Grupo experimental } & \multirow{2}{*}{$\begin{array}{c}\text { Grupo } \\
\text { control }\end{array}$} \\
\hline & & Pre-Post & Pre-Seg1 & Pre-Seg2 & \\
\hline \multirow{2}{*}{ Ansiedad } & Z & -3.839 & -3.204 & -4.019 & .000 \\
\hline & Sig. & .000 & .001 & .000 & 1.000 \\
\hline \multirow{2}{*}{ Depresión } & $Z$ & -3.495 & -3.735 & -3.924 & -.561 \\
\hline & Sig. & .000 & .000 & .000 & .575 \\
\hline \multirow{2}{*}{ Sobrecarga } & $\mathrm{Z}$ & -4.021 & -4.027 & NA & -.207 \\
\hline & Sig. & .000 & .000 & NA & .836 \\
\hline
\end{tabular}

Se calculó el tamaño del efecto mediante la prueba $d$ de Cohen y el porcentaje del cambio, encontrando que en depresión y sobrecarga el tamaño del efecto fue "grande", "mediano" en ansiedad y con una disminución grande en todas las variables (Tabla 4).

Tabla 4. Tamaño del efecto $d$ de Cohen y porcentaje de cambio.

\begin{tabular}{|l|c|c|c|c|}
\cline { 1 - 1 } \multicolumn{1}{|c|}{ Escalas } & \multirow{2}{*}{$\boldsymbol{d}$ de Cohen } & & $\begin{array}{l}\text { Porcentaje } \\
\text { de cambio }\end{array}$ & \multicolumn{1}{|c}{} \\
\hline Ansiedad & 0.53 & Efecto mediano & 43 & Disminución grande \\
\hline Depresión & 1.09 & Efecto grande & 37 & Disminución grande \\
\hline Sobrecarga & 0.92 & Efecto grande & 36 & Disminución grande \\
\hline
\end{tabular}




\section{DISCUSIÓN}

Los resultados del estudio en cuanto al perfil del cuidador son congruentes con los reportados en la literatura (Egea y Sánchez, 2008; Lara, González y Blanco, 2008). La mayoría de los familiares cuidadores fueron mujeres las cuales proporcionaban cuidado constante a su familiar, habitaban en la misma casa del paciente y tenían una edad promedio de 44 años (Espín, 2011; Roca et al., 2000).

En los análisis univariados se puede observar que los familiares cuidadores que participaron en el estudio mostraban síntomas de ansiedad, depresión y sobrecarga. Al sobrevenir la ERC, las personas que la padecen deben modificar su estilo de vida y afrontar problemas que aumentan su complejidad, según la fase de la enfermedad y los procesos terapéuticos en los que se encuentren (Caballero, Pérez, Herrera, Manrique y Sánchez-Sosa, 2012). Además, el familiar que apoya al enfermo se enfrenta también a la dificultad de adaptarse ante los acontecimientos estresantes que aparecen en las diversas etapas de la enfermedad, por lo que necesita atención y apoyo para reducir o prevenir las consecuencias o las implicaciones relacionadas con su rol de cuidador.

En cuanto a los niveles de ansiedad, depresión y sobrecarga de los cuidadores, se obtuvieron reducciones significativas en el grupo de intervención, en comparación con el grupo control, siendo grande el efecto de la intervención. Estos hallazgos indican que la intervención basada en el modelo de solución de problemas fue efectiva para reducir la sintomatología (Otero et al., 2014; Vázquez et al., 2013).

Se encontró asimismo que los efectos de la intervención basada en el citado modelo se man- tuvieron a corto plazo, es decir, a los dos meses después del tratamiento (Vázquez et al., 2013), a pesar de que el temor ante la pérdida del familiar es persistente en los cuidadores de pacientes con enfermedad renal debido a las complicaciones en las últimas etapas y tratamientos. Finalmente, respecto a la sobrecarga, se halló evidencia de la eficacia del programa (Sherwood et al., 2012; Vázquez et al., 2010), incluso a un mes después de la intervención.

Por lo anterior, este tipo de intervenciones breves puede ser eficaz en el corto plazo para reducir la sobrecarga y las reacciones emocionales que son producto de los distintos estímulos estresores a los que se enfrenta el proveedor del cuidado; la razón es que la modalidad de terapia en solución de problemas puede dotar de conocimientos y habilidades a los cuidadores para resolver una considerable gama de problemas, como los propios del rol del cuidador, los económicos y la falta de apoyo de la familia y de las instituciones, entre otros.

El referido tipo de programas de intervención no había sido aplicado anteriormente en cuidadores de pacientes con ERC, por lo que el presente estudio es pionero en el área y abre la posibilidad de seguir trabajando sistemáticamente con esta población. Para ello, se sugiere agregar técnicas de manejo emocional que complementen el programa debido a que los pacientes se encuentran en estado terminal y las complicaciones aumentan la probabilidad de muerte. Algunas sugerencias metodológicas consisten en emplear un diseño experimental, seleccionar a los participantes de manera aleatoria y aumentar el número de ensayos para generalizar los resultados.

\section{REFERENCIAS}

Amato, D., Álvarez A., C., Castañeda L., R., Rodríguez, E., Ávila D., M., Arreola, F., Gómez, A., Ballesteros, H., Becerril, R. y Paniagua, R. (2005). Prevalence of chronic kidney disease in an urban Mexican population. Kidney International, 68, 11-17. doi: 10.1111/j.1523-1755.2005.09702.x.

Archury, D., Castaño, H., Gómez, L. y Guevara, N. (2011). Calidad de vida de los cuidadores de pacientes con enfermedades crónicas con parcial dependencia. Investigación en Enfermería: Imagen y Desarrollo, 13(1), $27-46$.

Armstrong, P. (2005). Thinking it through: women, work and caring in the new millennium. Medicine, 19(4), $266-271$.

Bañobre, A., Vázquez, J., Outeiriño, S., Rodríguez, M., González, M. y Graña, J. (2005). Efectividad de la intervención educativa en cuidadores de pacientes dependientes en diálisis y valoración de la carga. Revista de la Sociedad Española de Enfermería Nefrológica, 8(2), 156-165. 
Barrera, L., Galvis, C., Moreno, M., Pinto, N., Pinzón, M., Romero, E. y Sánchez, B. (2006). La habilidad de cuidado de los cuidadores familiares de personas con enfermedad crónica. Investigación y Educación en Enfermería, 26, 36-46.

Beck, A.T. y Steer, R.A. (1993a). Beck Anxiety Inventory Manual. San Antonio, TX: Psychological Corporation.

Beck, A.T. y Steer, R.A. (1993b). Beck Depression Inventory. Manual. San Antonio, TX: The Psychological Corporation.

Borroto, G., Almeida, J., Lorenzo, A., Alfonso, F. y Guerrero, C. (2007). Percepción de la calidad de vida por enfermos sometidos a tratamientos de hemodiálisis o trasplante renal: Estudio comparativo. Revista Cubana de Medicina, 46(3). Recuperado de http://scielo.sld.cu/scielo.php?script=sci_arttextypid=S0034-75232007000300004ylng=esytlng=es (8 de agosto de 2017).

Bustos, V. (2006). Habilidad del cuidador y funcionalidad de la persona cuidada. Aquichan, 6(1), 137-147.

Caballero, N., Pérez, I., Herrera, M., Manrique, M. y Sánchez-Sosa, J. (2012). Efectos de una intervención cognitivo-conductual sobre la adhesión terapéutica y regulación emocional en pacientes con enfermedades gastrointestinales. Psicología y Salud, $22(2), 257-273$.

Cameron, J., Shin, J., Williams, D. y Stewart, D. (2004). A brief problem-solving intervention for family caregivers to individuals with advanced cáncer. Journal of Psychosomatic Research, 57(2), 137-143.

Centro Nacional de Equidad de Género y Salud Reproductiva de la Secretaría de Salud y Centro Oncológico Integral Diana Laura Riojas de Colosio del Hospital Médica Sur (2006). Manual de apoyo para personas que brindan cuidado a otras. México: Secretaría de Salud.

D’Zurilla, T. (1993). Terapia de resolución de conflictos: competencia social, un nuevo enfoque en la intervención clínica. Bilbao: Desclée de Brouwer.

De la Cuesta, C. (2004). Construir un mundo para el cuidado. Revista Rol de Enfermería, 27(12), 843-851.

Demiris, G., Parker, O., Washington, K., Fruehling, L., Haggarty-Robbins, D., Doorenbos, A. y Berry, D. (2010). A problem solving intervention for hospice caregivers: A pilot study. Journal of Palliative Medicine, 13(8), 1005-1011.

Domínguez G., M. (2012). Tercer informe del proyecto de evaluación de un modelo estructural de bienestar subjetivo en cuidadores familiares de adultos mayores. Manuscrito inédito. México: Consejo Nacional de Ciencia y Tecnología.

Ducharme, C., Lévesque, L., Lachance, M., Kergoat, J., Legault, J., Beaudet, M. y Zarit, H. (2011). "Learning to become a family caregiver". Efficacy of an intervention program for caregivers following diagnosis of dementia in a relative. The Gerontologist, 51(4), 484-494.

Egea, P. y Sánchez C., P. (2008). Programas e intervenciones de apoyo a los cuidadores informales en España. Gerokomos, 19(1), 9-15.

Elliot, T., Berry, J. y Grant, J. (2009). Problem-solving training for family caregivers of women with disabilities: A randomized clinical trial. Behaviour Research and Therapy, 47, 548-558.

Espín, A. (2009). "Escuela de Cuidadores" como programa psicoeducativo para cuidadores informales de adultos mayores con demencia. Revista Cubana de Salud Pública, 35(2). Recuperado http://scielo.sld.cu/scielo.php?script=sci_arttextypi$\mathrm{d}=$ S0864-34662009000200019ylng=esytlng=es (8 de agosto de 2017).

Espín, A. (2011). Eficacia de un programa psicoeducativo para cuidadores informales de adultos mayores con demencia. Revista de la Facultad de Salud, 3(1), 9-19.

Fernández, O. (2006). Calidad de vida de los cuidadores de jóvenes que viven en situación de enfermedad crónica en la ciudad de Ibagué. Tesis inédita de maestría. Bogotá: Universidad Nacional de Colombia.

Ferré G., C., Sevilla C., M., Boqué C., M., Aparicio C., M., Valdivieso L., A. y Lleixá F., M. (2012). Efectividad de la técnica de resolución de problemas aplicada por enfermeras: disminución de la ansiedad y la depresión en cuidadoras familiares. Atención Primaria, 44(12), 695-701.

Flórez T., I., Montalvo P., A., Herrera L., A. y Romero M., E. (2010). Afectación de los bienestares en cuidadores de niños y adultos con enfermedad crónica. Revista de Salud Pública, 12(5), 754-764.

Guerrero, L., Ramos, R., Alcolado, A., López, M., Pons, J. y Quesada, M. (2008). Programa de intervención multidisciplinaria para cuidadores de pacientes en atención domiciliaria. Gaceta Sanitaria, 22(5), 457-460.

Instituto Nacional de Estadística y Geografía (2013). Enfermedades crónicas. México: INEGI. Recuperado de http://www.inegi. org.mx/inegi/contenidos/espanol/prensa/Contenidos/estadisticas/2013/adultos8.pdf.

Jurado, S., Villegas, M.E., Méndez, L., Rodríguez, F., Loperena, V. y Varela, R. (1998). La estandarización del Inventario de Depresión de Beck para los residentes de la Ciudad de México. Salud Mental, 21, 26-31.

Ko, C., Malcarne, V., Varni, J., Roesch, S., Banthia, R., Greenbergs, H. y Robins, G. (2005). Problem-solving and distress in prostate cancer patients and their spousal caregivers. Support Care Cancer, 13, 367-374.

Lara, G., González, A. y Blanco, L. (2008). Perfil del cuidador: sobrecarga y apoyo familiar e institucional del cuidador primario en el primer nivel de atención. Revista de Especialidades Médico-Quirúrgicas, 13(4), 159-166.

Losada B., A. y Montorio, I. (2005). Pasado, presente y futuro de las intervenciones psicoeducativas para cuidadores familiares de personas mayores dependientes. Revista Española de Geriatría y Gerontología, 40, 30-39.

Martín C., M., Ballesteros R., J., Domínguez P., A., Muñoz H., P. y Gonzáles F., E. (2014). Intervenciones en el cuidador del enfermo con demencia. Actas Españolas de Psiquiatría, 42(6), 300-314. 
Meyers, J., Carducci, M., Loscalzo, J., Linder, J., Greasby, T. y Beckett, A. (2011). Effects of a problem-solving intervention (COPE) on quality of life for patients with advanced cancer on clinical trials and their caregivers: simultaneous care educational intervention (SCEI): linking palliation and clinical trials. Journal of Palliative Medicine, 14(4), 465-473.

Montero, X., Jurado, S., Robles, R., Aguilar, J., Figueroa, C. y Méndez, J. (2012). Carga en cuidadores primarios informales de niños con cáncer: efectos de una intervención cognitivo-conductual. Revista Latinoamericana de Medicina Conductual, 2(2), 118-124.

Nobili, A., Riva, E., Tettamanti, M., Lucca, U., Liscio, M., Petrucci, B. y Porro, G.S. (2004). The effect of a structured intervention on caregivers of patients with dementia and problem behaviors: A randomized controlled pilot study. Alzheimer Disease and Associated Disorders, 18, 75-82.

Organización Mundial de la Salud (2017). Enfermedades no transmisibles. Ginebra: OMS. Recuperado de http://www.who.int/ mediacentre/factsheets/fs355/es/.

Otero, P., Vázquez, F., Ferraces, M., Blanco, V. y Torres, A. (2014). Prevención de la depresión en cuidadoras no profesionales: relación entre habilidades de solución de problemas y síntomas depresivos. Clínica y Salud, 26(1), 1-7.

Rangel D., N., Ascencio H., L., Ornelas M., R., Allende P., S., Landa R., E. y Sánchez-Sosa, J.J. (2013). Efectos de la solución de problemas sobre los comportamientos de autocuidado de cuidadores de pacientes oncológicos en fase paliativa: un estudio piloto. Psicooncología, 9(2/3), 365-376.

Robles, R., Varela, R., Jurado, S. y Páez, F. (2001). Versión mexicana del Inventario de Ansiedad de Beck: propiedades psicométricas. Revista Mexicana de Psicología, 18(2), 211-218.

Roca, M., Úbeda, I., Fuentelsaz, C., López, R., Pont, A., García, L. y Pedreny, R. (2000). Impacto del hecho de cuidar en la salud de los cuidadores familiares. Atención Primaria, 26, 53-67.

Seidmann, S., Bail, V., Acrich, 1. y Stéfani, D. (2001). Cuidando a un familiar enfermo. Una aproximación cualitativa. $I X$ Anuario de Investigaciones de la Facultad de Psicología (pp. 96-99): Buenos Aires: UBA.

Sherwood, R., Given, A., Given, W., Sikorskii, A., You, M. y Prince, J. (2012). The impact of a problem-solving intervention on increasing caregiver assistance and improving caregiver health. Support Care Cancer, 20(9), 1937-1947.

Spielberger, C.D. y Díaz-Guerrero, R. (1975). IDARE: Inventario de ansiedad rasgo-estado. México: El Manual Moderno.

Tong, W., Yip, A., Liu, J. y McMaster, T. (2016). The effectiveness of manual-guided, problem-solving-based self-learning programme for family caregivers of people with recent-onset psychosis: A randomised controlled trial with 6-month follow-up. International Journal of Nursing Studies, 59, 141-155.

Vázquez F., L., Otero, P., Torres, A., Hermida, E., Blanco, V. y Díaz, O. (2013). A brief, problem-solving, indicated-prevention intervention for depression in nonprofessional caregivers. Psicothema, 25, 87-92.

Vázquez, F., Otero, P., López, M., Blanco, V. y Torres, A. (2010). Un programa breve basado en la solución de problemas para la prevención de la depresión en cuidadores informales de pacientes con demencia: un estudio piloto. Clínica y Salud, 21(1), 59-76. doi: 10.5093/c12010v21n1a6.

Vega A., O., Mendoza T., M., Ureña M., M. y Villamil, W. (2008). Efecto de un programa educativo en la habilidad de cuidado de los cuidadores familiares de personas en situación crónica de enfermedad. Ciencia y Cuidado, 5(1), 5-19.

Velásquez, V., López, L., López, H., Cataño, N. y Muñoz, E. (2011). Efecto de un programa educativo para cuidadores de personas ancianas: una perspectiva cultural. Revista de Salud Pública, 13(3), 458-469.

Vinyoles, E., Copetti, S., Cabezas, C., Megido, M., Espinàs, J., Vila, J., Montella, N., Martínez, A., Argimón, J. y Grupo de Estudio CUIDA'L (2001). Cuida'1: un ensayo clínico que evaluará la eficacia de una intervención en cuidadores de pacientes demenciados. Atención Primaria, 27(1), 49-53.

Zambrano, R. y Ceballos, P. (2007). Síndrome de carga del cuidador. Revista Colombiana de Psiquiatría, 36(1), $26-39$. 